\title{
Revista Colombiana de

\section{Guía basada en la evidencia para el manejo perioperatorio de la anticoagulación oral con warfarina en pacientes con alto riesgo embólico que serán llevados a implante de dispositivos de estimulación cardiaca}

\author{
Alejandro Olaya ${ }^{\mathrm{a}, *}$, Hernán Calvo ${ }^{\mathrm{b}}$, Catalina Pinzón ${ }^{\mathrm{b}}$, Magda Alba $^{\mathrm{c}}$, Magda Cepeda $^{\mathrm{c}}$, \\ John Liévano ${ }^{\mathrm{b}}$, María H. Solano ${ }^{\mathrm{d}}$ y Guillermo Mora ${ }^{\mathrm{e}}$
}

a Programa de especialización en Cardiología, Fundación Universitaria de Ciencias de la Salud. Servicio de Electrofisiología, Hospital Cardiovascular del Niño de Cundinamarca, Electrofisiología Fundación Santa Fe, Bogotá, Colombia

${ }^{\mathrm{b}}$ Fundación Universitaria de Ciencias de la Salud, Bogotá, Colombia

c División de investigaciones, Fundación Universitaria de Ciencias de la Salud, Bogotá, Colombia

d Programa de especialización en Hematología, Fundación Universitaria de Ciencias de la Salud, Bogotá, Colombia

e Electrofisiología, Universidad Nacional de Colombia. Fundación Santa Fe, Bogotá, Colombia

Recibido el 16 de mayo de 2014; aceptado el 17 de octubre de 2014

Disponible en Internet el 19 de marzo de 2015

\section{PALABRAS CLAVE Anticoagulación; Heparina; Marcapasos; Desfibrilador; Tratamiento anticoagulante}

\begin{abstract}
Resumen
Introducción: El manejo perioperatorio de la anticoagulación crónica representa dificultad para los médicos que realizan implantes de dispositivos de estimulación cardíaca, quienes utilizan la terapia puente con heparinas con la intención de disminuir el riesgo de sangrado. Sin embargo, este tratamiento es controversial.

Objetivo: Generar recomendaciones basadas en evidencia sobre el tratamiento perioperatorio de la anticoagulación de los pacientes con riesgo embólico moderado y alto, que requieren anticoagulación oral con warfarina y serán llevados a implante de dispositivos de estimulación cardíaca.

Métodos: Se realizó una búsqueda de la literatura para identificar guías de práctica clínica basadas en evidencia, las cuales se calificaron con la herramienta AGREE II. Se actualizó la evidencia desde el 1 de enero de 2009 hasta el 30 de noviembre de 2013, incluidas revisiones sistemáticas y experimentos clínicos. Los estudios se calificaron con los instrumentos GRADE y SIGN. Se generaron recomendaciones con metodología GRADE, llevadas a consenso formal de expertos.
\end{abstract}

\footnotetext{
* Autor para correspondencia.

Correo electrónico: aolaya@fucsalud.edu.co (A. Olaya).
} 
Resultados: Las guías SIGN y CHEST tuvieron la mejor calificación con AGREE II. Con la nueva evidencia disponible proveniente de tres revisiones sistemáticas y dos experimentos clínicos, se generó recomendación fuerte a favor de continuar la anticoagulación oral con warfarina durante el perioperatorio de implante de dispositivos de estimulación cardíaca en pacientes con riesgo embólico moderado y alto.

Conclusiones: A partir de la nueva evidencia cambia la recomendación propuesta por las guías SIGN y CHEST. La alternativa de continuar la terapia con warfarina a cambio de realizar terapia puente, debe considerarse en la práctica clínica.

(c) 2014 Sociedad Colombiana de Cardiología y Cirugía Cardiovascular. Publicado por Elsevier España, S.L.U. Este es un artículo Open Access bajo la licencia CC BY-NC-ND (http://creativecommons.org/licenses/by-nc-nd/4.0/).

\section{KEYWORDS}

Anticoagulation;

Heparin;

Pacemakers;

Defibrillator;

Anticoagulant

therapy
Evidence-based guidelines for the perioperative management of oral anticoagulation with warfarin in patients scheduled for implantation of a cardiac electronic device

\begin{abstract}
Introduction: The perioperative management of chronic anticoagulation concerns all physicians involved in the implantation of cardiac electronic devices (either pacemakers and cardioverterdefibrillators). Switching from warfarin to low molecular weight heparins (LMWH) has been performed to reduce the risk of bleeding. However, this approach has been shown to be controversial.

Objective: To generate evidence-based recommendations on the perioperative management of oral anticoagulation with warfarin in patients who will undergo the implantation of a cardiac electronic device.

Methods: A literature search was performed to identify evidence-based clinical practice guidelines, which were assessed using the AGREE II instrument. The guidelines were updated with systematic reviews and randomized clinical trials published from February 2009 to November 2013 comparing the continuation of warfarin versus switching to a LMWH or withholding warfarin. These studies were analyzed using the SIGN and GRADE scales to assess methodological quality. Recommendations were put forward through an expert panel.

Results: The guidelines with highest methodological quality were the SIGN (Scottish Intercollegiate Guidelines Network) guideline published in June 2013 and the CHEST guideline published in February 2012. The update included three systematic reviews and two randomized clinical trials. The continuation of warfarin for the perioperative management of implantable cardiac electronic devices in patients with moderate and high risk is recommended.

Conclusion: Based on the new evidence changes the recommendation given by the CHEST SIGN and guides. The alternative of continued therapy with warfarin in exchange for performing therapy bridge, should be considered in clinical practice.

(C) 2014 Sociedad Colombiana de Cardiología y Cirugía Cardiovascular. Published by Elsevier España, S.L.U. This is an open access article under the CC BY-NC-ND license (http://creativecommons.org/licenses/by-nc-nd/4.0/).
\end{abstract}

\section{Introducción}

Se estima que cada año se implantan en todo el mundo 1,25 millones de marcapasos y 410.000 cardioversoresdesfibriladores $^{1}$. Entre el 14 y el $35 \%$ de los pacientes que reciben este tipo de dispositivos requiere anticoagulación oral con warfarina a largo plazo ${ }^{2}$, dado que son clasificados en riesgo moderado o alto para tromboembolia arterial, con un compromiso a un año del 5 al $10 \%$ y mayor a $10 \%$, respectivamente ${ }^{3}$.

De otro lado, el manejo perioperatorio de la anticoagulación crónica representa una dificultad considerable para los médicos que practican este tipo de implantes, quienes utilizan la terapia puente con heparinas con la intención de minimizar el riesgo de tromboembolia arterial y de trombosis recurrente en pacientes con tromboembolia venosa previa, así como también de disminuir el riesgo de sangrado ${ }^{3}$. Sin embargo, el uso de terapia puente con heparinas en el periodo perioperatorio genera controversia, pues no es claro su beneficio en la reducción del riesgo de hematoma del bolsillo clínicamente significativo.

Hoy las guías de práctica clínica ${ }^{3}$ sugieren el uso de terapia puente con heparinas en el manejo perioperatorio de pacientes con alto riesgo de tromboembolia venosa que serán llevados a implante de dispositivos cardíacos. Pese a ello, nueva evidencia sugiere que se benefician de la no interrupción de la anticoagulación con warfarina, en comparación con la realización de terapia puente con heparinas, en vista de que se observa reducción en el riesgo de sangrado, sin incremento del riesgo de eventos tromboembólicos. 
El objetivo de esta guía es generar recomendaciones sobre el manejo perioperatorio de la anticoagulación de pacientes, quienes, por su riesgo embólico moderado y alto, requieren anticoagulación oral con warfarina y serán llevados a implante de dispositivos de estimulación cardíaca (marcapasos o cardiodesfibriladores implantables).

\section{Materiales y métodos}

La guía está dirigida al personal médico (cardiólogos, electrofisiólogos, hemodinamistas, hematólogos, anestesiólogos, especialistas en Medicina Interna, Medicina de Urgencias y Medicina Familiar) y al personal de salud que asiste pacientes anticoagulados con warfarina de manera crónica, y que serán llevados a implante de dispositivos de estimulación cardíaca.

Excede el alcance de esta guía, generar recomendaciones sobre la efectividad y seguridad de otras terapias anticoagulantes orales (dabigatrán, rivaroxabán y apixabán).

Se definió la estrategia PICO para realizar la búsqueda de la literatura:

- Población: pacientes en terapia crónica de anticoagulación oral con warfarina en riesgo alto y moderado de eventos, que serán llevados a implante de dispositivos cardíacos (marcapasos o cardiodesfibriladores).

- Intervención: warfarina ininterrumpida o no uso de warfarina.

- Comparador: terapia puente con heparinas o suspensión de la anticoagulación oral.

- Desenlaces: eventos tromboembólicos y hematoma del bolsillo.

Estos desenlaces fueron calificados como críticos, de acuerdo con la herramienta GRADE ${ }^{4}$.

Dos evaluadores realizaron la identificación de las guías de práctica clínica. El criterio de selección fueron guías publicadas en los últimos cinco años, en idioma español o inglés, que presentaran un proceso sistemático para la búsqueda de evidencia y la generación de las recomendaciones. Se buscaron bases de datos de National Guideline Clearinghouse, National Institute for Clinical Excellence, New Zealand Guidelines Group, HTA: Health Technology Assessment database, National Institute and Medical Research Council, Guía Salud y en los buscadores MEDLINE- PubMed, TripDatabase, National Electronic Library for Health, MEDCARIB-Literatura del Caribe en Ciencias de la Salud, LILACS, WHOLIS - Sistema de Información de la Biblioteca de la OMS, PAHO- Catálogo de la Biblioteca Sede de la OPS y Fisterra.

Se utilizaron los términos MeSH: «anticoagulants» OR «warfarin» «heparin, low-molecular-weight» AND «perioperative care» OR «perioperative period» AND «thromboembolism» AND «pacemaker, artificial» OR «implantable cardioverter-defibrillator» OR «elective surgical procedures».

Para la valoración de la calidad metodológica, cuatro investigadores (AOS, HCM, CPR y MAS) utilizaron el instrumento AGREE II (Appraisal of Guidelines for Research \& Evaluation $)^{5}$. Se seleccionaron las guías con mayor calidad para el proceso de adaptación. A partir de la información de las guías seleccionadas se definió una nueva estrategia de búsqueda para la actualización de la evidencia, que incluyó revisiones sistemáticas de la literatura y experimentos clínicos publicados entre el 1 de enero de 2009 y 30 de octubre 2013. Se utilizó la herramienta SIGN (Scottish Intercollegiate Guidelines Network) ${ }^{6}$ y el software GRADE pro 3.6 para jerarquizar la calidad de la evidencia y la fuerza de las recomendaciones ${ }^{4}$.

Todos los participantes del grupo desarrollador firmaron la declaración de conflicto de intereses.

La formulación de las recomendaciones se realizó en consenso formal de expertos en Cardiología, Hemodinamia, Hematología, Medicina Interna, Medicina de Urgencias y Epidemiología, utilizando un sistema de votación electrónico, discusión abierta y nuevamente votación electrónica hasta obtener el acuerdo por mayoría.

Al momento del sometimiento a publicación de esta guía se propuso la estrategia de divulgación de estos resultados a través de las sociedades científicas.

\section{Resultados}

La figura 1 muestra el proceso de selección de guías de práctica clínica. Se identificaron 59 guías de las cuales cinco cumplían los criterios de elegibilidad ${ }^{3,7-10}$. La guía $\mathrm{CHEST}^{3}$ de 2012 y la guía escocesa SIGN $129^{10}$ fueron seleccionadas para la adaptación (tabla 1 ). La recomendación de la guía SIGN 129 no está dirigida específicamente al procedimiento de implante de dispositivos cardíacos, sino que hace referencia a pacientes que serán sometidos a diferentes procedimientos invasivos, y a la vez sugiere la individualización de cada caso con base en el riesgo de hemorragia y trombosis y en la naturaleza del procedimiento que se realizará ${ }^{10}$. Cita una revisión sistemática que incluyó ocho estudios de anticoagulación en procedimientos de implante de cardiodesfibriladores o marcapasos mostrando bajas tasas de tromboembolia tanto con la terapia oral ininterrumpida como con el uso de heparina en el perioperatorio; sin embargo, reporta que la terapia puente con heparina mostró mayor frecuencia de sangrado respecto a la continuación de la terapia oral ${ }^{11}$. La guía $\mathrm{CHEST}^{3}$, entre tanto, recomienda la terapia puente en pacientes con válvulas mecánicas, fibrilación auricular o alto riesgo de tromboembolia. Estas son recomendaciones basadas en evidencia de baja o muy baja calidad (2 C).

\section{Actualización de la evidencia}

Se encontraron tres metaanálisis ${ }^{12-14}$ y dos ensayos clínicos $^{2,15}$ publicados entre el 1 de enero de 2009 y 30 de octubre de 2013; ninguna de estas publicaciones había sido incluida en las guías $\mathrm{CHEST}^{3}$ y $\mathrm{SIGN}^{10}$ y aportan nueva evidencia. En la tabla 2 se resumen los hallazgos de ambos estudios.

El metaanálisis llevado a cabo por Ghanbari et al. ${ }^{14}$ en 2012, obtuvo una calificación de calidad moderada por riesgo de sesgo. Este estudio incluyó población de riesgo embólico moderado y alto, y concluyó que la terapia con warfarina ininterrumpida se asocia con un riesgo significativamente menor de complicaciones hemorrágicas postoperatorias en comparación con la terapia puente con 


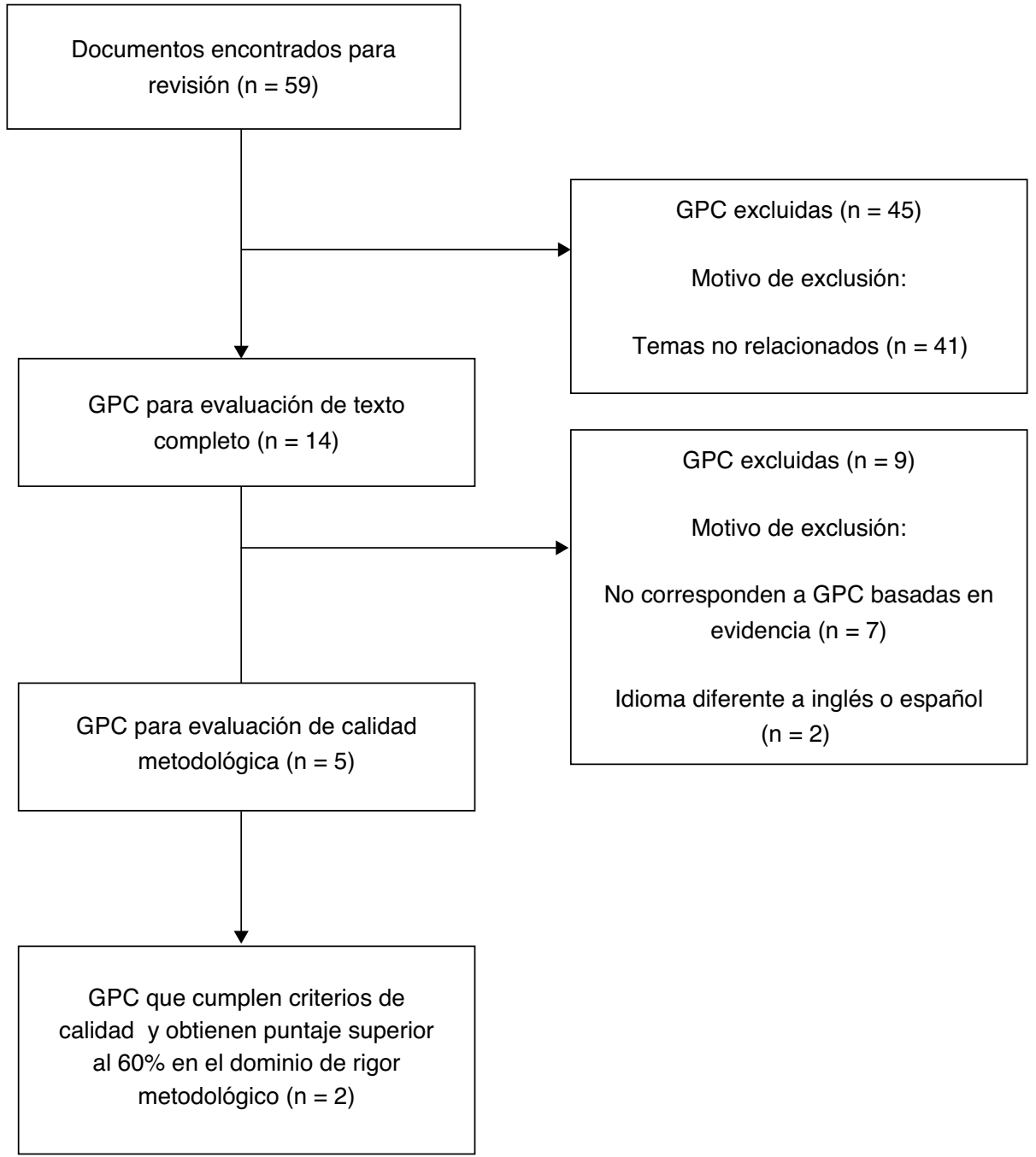

Figura 1 Flujograma de selección de guías de practicá clínica (GPC).

Tabla 1 Recomendaciones para el manejo de la anticoagulación oral

\begin{tabular}{|c|c|c|}
\hline & $\begin{array}{l}\text { Guía CHEST } 2012^{3} \\
\text { Manejo perioperatorio de la terapia } \\
\text { antitrombótica }\end{array}$ & $\begin{array}{l}\text { Guía Escocesa SIGN 129. } 2013^{10} \\
\text { Antitrombóticos: indicaciones y manejo }\end{array}$ \\
\hline Recomendaciones & $\begin{array}{l}\text { En pacientes con válvulas mecánicas, } \\
\text { fibrilación auricular o tromboembolia venosa y } \\
\text { alto riesgo de eventos tromboembólicos, se } \\
\text { sugiere realizar terapia puente con heparinas } \\
\text { en lugar de no hacerlo durante el tiempo de } \\
\text { interrupción de los antagonistas de la vitamina } \\
\mathrm{K} \\
\text { En pacientes con válvulas mecánicas, } \\
\text { fibrilación auricular o tromboembolia venosa y } \\
\text { riesgo moderado de eventos tromboembólicos, } \\
\text { la elección de realizar terapia puente o no } \\
\text { hacerlo, se basa en la evaluación individual del } \\
\text { paciente y de los factores relacionados con la } \\
\text { cirugía }\end{array}$ & $\begin{array}{l}\text { La decisión relacionada con la interrupción del } \\
\text { tratamiento con antagonistas de vitamina K } \\
\text { para otros procedimientos quirúrgicos e } \\
\text { invasivos, y sobre si la terapia puente es } \\
\text { recomendable, debe hacerse sobre una base } \\
\text { individual y depende de los riesgos } \\
\text { establecidos de hemorragia asociada a la } \\
\text { continuación de la anticoagulación o de } \\
\text { trombosis relacionados con la interrupción de } \\
\text { la misma, así como de la naturaleza del } \\
\text { procedimiento propuesto }\end{array}$ \\
\hline $\begin{array}{l}\text { Fecha de } \\
\text { búsqueda }\end{array}$ & Enero de 1970 a enero de 2010 & $\begin{array}{l}\text { 2003-2009. } \\
\text { Actualización junio de } 2013\end{array}$ \\
\hline
\end{tabular}


Tabla 2 Resumen de la evidencia

\begin{tabular}{|c|c|c|c|c|c|c|c|c|c|c|c|}
\hline \multirow[b]{2}{*}{ Autor } & \multirow[b]{2}{*}{ Desenlace } & \multicolumn{4}{|c|}{ Evaluación de la calidad } & \multicolumn{3}{|c|}{$n$} & \multirow{2}{*}{$\begin{array}{l}\text { Efecto } \\
\text { Relativo } \\
\text { (IC 95\%) }\end{array}$} & \multirow{2}{*}{ Calidad } & \multirow{2}{*}{ Importancia } \\
\hline & & $\begin{array}{l}\text { Número } \\
\text { de } \\
\text { estudios }\end{array}$ & Diseño & $\mathrm{n}$ & $\begin{array}{l}\text { Riesgo } \\
\text { de sesgo }\end{array}$ & $\begin{array}{l}\text { Suspender } \\
\text { Anticoagulación }\end{array}$ & $\begin{array}{l}\text { Warfarina } \\
\text { Ininterrumpida }\end{array}$ & $\begin{array}{l}\text { Terapia } \\
\text { puente con } \\
\text { heparina }\end{array}$ & & & \\
\hline \multirow[t]{2}{*}{$\begin{array}{l}\text { Ghanbari } \\
\text { H. } 2012 \\
(14)\end{array}$} & $\begin{array}{l}\text { Hemorragia } \\
\text { post } \\
\text { operatoria }\end{array}$ & 8 & $\begin{array}{l}\text { Metaanálisis: } \\
\text { Estudios } \\
\text { observacionales } \\
\text { ( } 5 \text { cohortes) } \\
\text { Experimentales } \\
\text { (3) }\end{array}$ & 2,321 & Serio & - & $40 / 953(4,2 \%)$ & $\begin{array}{l}78 / 695 \\
(11,2 \%)\end{array}$ & $\begin{array}{l}\text { OR } 0,30 \\
(0,18 \mathrm{a} \\
0,50)\end{array}$ & Moderada & Crítica \\
\hline & $\begin{array}{l}\text { Eventos } \\
\text { trom- } \\
\text { boembóli- } \\
\text { cos }\end{array}$ & 4 & $\begin{array}{l}\text { Metaanálisis: } \\
\text { estudios } \\
\text { observacionales } \\
\text { ( } 3 \text { cohortes) } \\
\text { experimentales } \\
\text { (1) }\end{array}$ & 1,286 & Muy serio & - & $2 / 716(0,27 \%)$ & $\begin{array}{l}3 / 570 \\
(0,52 \%)\end{array}$ & $\begin{array}{l}\text { OR } 0,65 \\
(0,14 \mathrm{a} \\
3,02)\end{array}$ & Baja & Crítica \\
\hline \multirow[t]{2}{*}{$\begin{array}{l}\text { Siegal D. } \\
2012^{a} \\
(12)\end{array}$} & Sangrado & 13 & $\begin{array}{l}\text { Metaanálisis } \\
\text { Estudios } \\
\text { observacionales } \\
\text { (13) }\end{array}$ & 7,095 & Muy serio & - & $102 / 5,160(2,0 \%)$ & $\begin{array}{l}217 / 1935 \\
(11,2 \%)\end{array}$ & $\begin{array}{l}\text { OR } 5,4(3,0 \\
\text { a } 9,74)\end{array}$ & Baja & Crítica \\
\hline & $\begin{array}{l}\text { Eventos } \\
\text { trom- } \\
\text { boembóli- } \\
\text { cos }\end{array}$ & 8 & $\begin{array}{l}\text { Metaanálisis } \\
\text { Estudios } \\
\text { observacionales } \\
\text { (8) }\end{array}$ & 5,188 & Muy serio & - & $32 / 3.497(0,91 \%)$ & $\begin{array}{l}19 / 1691 \\
(1,12 \%)\end{array}$ & $\begin{array}{l}\text { OR } 0,80 \\
(0,42-1,54)\end{array}$ & Baja & Crítica \\
\hline \multirow[t]{2}{*}{$\begin{array}{l}\text { Bernard } \\
\text { M. } 2012^{\mathrm{C}} \\
(13)\end{array}$} & $\begin{array}{l}\text { Sangrado } \\
\text { mayor }\end{array}$ & 11 & $\begin{array}{l}\text { Metaanálisis } \\
\text { Estudios } \\
\text { observacionales } \\
\text { (9) Estudios } \\
\text { experimentales } \\
\text { (2) }\end{array}$ & 1,630 & Muy serio & - & $5 / 1,079(0,46 \%)$ & $\begin{array}{l}11 / 551 \\
(2,0 \%)\end{array}$ & $\begin{array}{l}\text { No } \\
\text { reportado }\end{array}$ & Muy baja & Crítica \\
\hline & $\begin{array}{l}\text { Sangrado } \\
\text { mayor }\end{array}$ & 5 & $\begin{array}{l}\text { Metaanálisis } \\
\text { Estudios } \\
\text { observacionales } \\
\text { Estudios } \\
\text { experimentales }\end{array}$ & 2,123 & Muy serio & $2 / 1044(0,2 \%)$ & $5 / 1,079(0,46 \%)$ & - & $\begin{array}{l}\text { No } \\
\text { reportado }\end{array}$ & Muy baja & Crítica \\
\hline
\end{tabular}


Tabla 2 (continuación)

\begin{tabular}{|c|c|c|c|c|c|c|c|c|c|c|c|}
\hline \multirow[b]{2}{*}{ Autor } & \multirow[b]{2}{*}{ Desenlace } & \multicolumn{4}{|c|}{ Evaluación de la calidad } & \multicolumn{3}{|c|}{$\mathrm{n}$} & \multirow{2}{*}{$\begin{array}{l}\text { Efecto } \\
\text { Relativo } \\
\text { (IC 95\%) }\end{array}$} & \multirow[t]{2}{*}{ Calidad } & \multirow[t]{2}{*}{ Importancia } \\
\hline & & $\begin{array}{l}\text { Número } \\
\text { de } \\
\text { estudios }\end{array}$ & Diseño & $\mathrm{n}$ & $\begin{array}{l}\text { Riesgo } \\
\text { de sesgo }\end{array}$ & $\begin{array}{l}\text { Suspender } \\
\text { Anticoagulación }\end{array}$ & $\begin{array}{l}\text { Warfarina } \\
\text { Ininterrumpida }\end{array}$ & $\begin{array}{l}\text { Terapia } \\
\text { puente con } \\
\text { heparina }\end{array}$ & & & \\
\hline & $\begin{array}{l}\text { Riesgo de } \\
\text { sangrado } \\
\text { (mayor y } \\
\text { menor) }\end{array}$ & 11 & $\begin{array}{l}\text { Metaanálisis } \\
\text { Estudios } \\
\text { observacionales } \\
\text { (11) }\end{array}$ & 1,630 & Muy serio & & $29 / 1,079(2,68 \%)$ & $\begin{array}{l}61 / 551 \\
(11,07 \%)\end{array}$ & $\begin{array}{l}\text { OR 5, } 3^{\mathrm{b}}(\mathrm{IC} \\
\text { Cred } 95 \% \\
3,4 \text { a } 8,5)\end{array}$ & Muy baja & Crítica \\
\hline & \multirow[t]{2}{*}{$\begin{array}{l}\text { Riesgo de } \\
\text { sangrado } \\
\text { (mayor y } \\
\text { menor) }\end{array}$} & 5 & Metaanálisis & 1,807 & Muy serio & $23 / 990(2,3 \%)$ & $18 / 817(2,2 \%)$ & - & $\begin{array}{l}\text { No } \\
\text { reportado }\end{array}$ & Muy baja & Crítica \\
\hline & & & $\begin{array}{l}\text { Estudios } \\
\text { observacionales } \\
\text { (4) estudio } \\
\text { experimental (1) }\end{array}$ & & & & & & & & \\
\hline & \multirow[t]{2}{*}{$\begin{array}{l}\text { Riesgo de } \\
\text { sangrado } \\
\text { (mayor y } \\
\text { menor) }\end{array}$} & 6 & Metaanálisis & 746 & Muy serio & - & $24 / 412(5,8 \%)$ & $\begin{array}{l}57 / 334 \\
(17,1 \%)\end{array}$ & $\begin{array}{l}\text { No } \\
\text { reportado }\end{array}$ & Muy baja & Crítica \\
\hline & & & $\begin{array}{l}\text { Estudios } \\
\text { observacionales } \\
\text { (4) estudio } \\
\text { experimental (2) }\end{array}$ & & & & & & & & \\
\hline & \multirow[t]{2}{*}{$\begin{array}{l}\text { Eventos } \\
\text { trom- } \\
\text { boembóli- } \\
\text { cos }\end{array}$} & 5 & Metaanálisis & 878 & Muy serio & - & $1 / 463(0,2 \%)$ & $2 / 415(0,5 \%)$ & $\begin{array}{l}\text { No } \\
\text { reportado }\end{array}$ & Muy baja & Crítica \\
\hline & & & $\begin{array}{l}\text { Estudios } \\
\text { observacionales } \\
\text { (4) estudio } \\
\text { experimental (1) }\end{array}$ & & & & & & & & \\
\hline & $\begin{array}{l}\text { Eventos } \\
\text { trom- } \\
\text { boembóli- } \\
\text { cos }\end{array}$ & 4 & $\begin{array}{l}\text { Metaanálisis } \\
\text { Estudios } \\
\text { observacionales } \\
\text { (3) } \\
\text { Experimentales } \\
\text { (1) }\end{array}$ & 1,765 & Muy serio & $5 / 977(0,5 \%)$ & $1 / 788(0,12 \%)$ & - & $\begin{array}{l}\text { No } \\
\text { reportado }\end{array}$ & Muy baja & Crítica \\
\hline
\end{tabular}




\begin{tabular}{|c|c|c|c|c|c|c|c|c|c|c|c|}
\hline \multirow[t]{5}{*}{$\begin{array}{l}\text { Birnie } \\
\text { DH. } 2013\end{array}$} & $\begin{array}{l}\text { Hematoma } \\
\text { clínica- } \\
\text { mente } \\
\text { impor- } \\
\text { tante }\end{array}$ & 1 & $\begin{array}{l}\text { Experimento } \\
\text { clínico }\end{array}$ & 681 & No & No evaluado & $12 / 343(3,5 \%)$ & $\begin{array}{l}54 / 338 \\
(16,0 \%)\end{array}$ & $\begin{array}{l}\text { RR } 0,19(0,1 \\
\text { a } 0,36)\end{array}$ & Alta & Crítica \\
\hline & $\begin{array}{l}\text { Hematoma } \\
\text { que } \\
\text { requiere } \\
\text { aumento } \\
\text { de } \\
\text { estancia } \\
\text { hospitala- } \\
\text { ria }\end{array}$ & & & & No & No evaluado & $4 / 343(1,2 \%)$ & $\begin{array}{l}16 / 338 \\
(4,7 \%)\end{array}$ & $\begin{array}{l}\text { RR } 0,24 \\
(0,08 \mathrm{a} \\
0,72)\end{array}$ & Alta & Crítica \\
\hline & $\begin{array}{l}\text { Hematoma } \\
\text { que } \\
\text { requiere } \\
\text { interrup- } \\
\text { ción de } \\
\text { anticoagu- } \\
\text { lación }\end{array}$ & & & & No & No evaluado & $11 / 343(3,2 \%)$ & $\begin{array}{l}48 / 338 \\
(14,2)\end{array}$ & $\begin{array}{l}\operatorname{RR} 0,2(0,1 \\
\text { a } 0,39)\end{array}$ & Alta & Crítica \\
\hline & $\begin{array}{l}\text { Hematoma } \\
\text { que } \\
\text { requiere } \\
\text { evacua- } \\
\text { ción }\end{array}$ & & & & $\begin{array}{l}\text { Otras limi- } \\
\text { taciones } \\
\text { por } \\
\text { tamaño de } \\
\text { muestra }\end{array}$ & No evaluado & $2 / 343(0,6 \%)$ & $9 / 338(2,7 \%)$ & $\begin{array}{l}\text { RR } 0,21 \\
(0,05 \text { a } 1,0)\end{array}$ & Moderada & Crítica \\
\hline & $\begin{array}{l}\text { Eventos } \\
\text { trom- } \\
\text { boembóli- } \\
\text { cos }\end{array}$ & & & & $\begin{array}{l}\text { Otras limi- } \\
\text { taciones } \\
\text { por } \\
\text { tamaño de } \\
\text { muestra }\end{array}$ & No evaluado & $2 / 343(0,6 \%)$ & $1 / 338(0,3 \%)$ & $\begin{array}{l}\text { RR no } \\
\text { reportado. } \\
\text { Valor } p \text { no } \\
\text { significativo }\end{array}$ & Moderada & Crítica \\
\hline
\end{tabular}


Tabla 2 (continuación)

\begin{tabular}{|c|c|c|c|c|c|c|c|c|c|c|c|}
\hline \multirow[b]{2}{*}{ Autor } & \multirow[b]{2}{*}{ Desenlace } & \multicolumn{4}{|c|}{ Evaluación de la calidad } & \multicolumn{3}{|c|}{$\mathrm{n}$} & \multirow{2}{*}{$\begin{array}{l}\text { Efecto } \\
\text { Relativo } \\
\text { (IC 95\%) }\end{array}$} & \multirow[t]{2}{*}{ Calidad } & \multirow[t]{2}{*}{ Importancia } \\
\hline & & $\begin{array}{l}\text { Número } \\
\text { de } \\
\text { estudios }\end{array}$ & Diseño & $\mathrm{n}$ & $\begin{array}{l}\text { Riesgo } \\
\text { de sesgo }\end{array}$ & $\begin{array}{l}\text { Suspender } \\
\text { Anticoagulación }\end{array}$ & $\begin{array}{l}\text { Warfarina } \\
\text { Ininterrumpida }\end{array}$ & $\begin{array}{l}\text { Terapia } \\
\text { puente con } \\
\text { heparina }\end{array}$ & & & \\
\hline \multirow[t]{3}{*}{$\begin{array}{l}\text { Juhani } \\
\text { Airaksinen } \\
2013^{d}\end{array}$} & $\begin{array}{l}\text { Cualquier } \\
\text { hematoma } \\
\text { del } \\
\text { bolsillo }\end{array}$ & 1 & $\begin{array}{l}\text { Experimento } \\
\text { clínico }\end{array}$ & 213 & $\begin{array}{l}\text { Otras limi- } \\
\text { taciones } \\
\text { por } \\
\text { tamaño de } \\
\text { muestra }\end{array}$ & $43 / 107(40,1 \%)$ & $35 / 106(33,0 \%)$ & No evaluado & $\begin{array}{l}\text { HR 0,86 } \\
(0,66-1,12)\end{array}$ & Moderada & Crítica \\
\hline & $\begin{array}{l}\text { Hematoma } \\
\text { mayor }\end{array}$ & & & & & $6 / 107(6,0 \%)$ & $6 / 106(6,0 \%)$ & No evaluado & $\begin{array}{l}\text { No } \\
\text { reportado }\end{array}$ & Moderada & Crítica \\
\hline & $\begin{array}{l}\text { Eventos } \\
\text { trom- } \\
\text { boembóli- } \\
\text { cos }\end{array}$ & & & & & $1 / 107$ & $0 / 106$ & No evaluado & $\begin{array}{l}\text { No } \\
\text { reportado ( } 0 \\
\text { eventos } \\
\text { grupo } \\
\text { warfarina } \\
\text { ininterrum- } \\
\text { pida) }\end{array}$ & Moderada & Crítica \\
\hline
\end{tabular}

* Ghanbari compara warfarina ininterrumpida con terapia puente. La medida de efecto OR representa reducción del riesgo de sangrado con warfarina ininterrumpida.

a Siegal compara terapia puente con no terapia puente, por tanto la medida de efecto representa incremento del riesgo de sangrado con terapia puente.

b Intervalo de credibilidad 95\%. Análisis bayesiano.

c Bernard. Estudios heterogéneos ya que no existe consenso entre los artículos respecto a la asignación de riesgo tromboembólico. Datos de seis estudios reportan que el $50,5 \%$ de los pacientes fueron clasificados en alto riesgo y el $17,2 \%$ en riesgo moderado. La mayoría de los estudios son observacionales.

d Juhani Airaksinen: protocolo suspender anticoagulación dos días. HR representa la comparación warfarina ininterrumpida vs. la interrupción de anticoagulación oral por dos días. Insuficiente tamaño de muestra para desenlaces analizados. Los autores declaran que debe ser considerado como análisis descriptivo y exploratorio pero no suficiente para concluir no inferioridad o superioridad de una terapia respecto a otra. 
heparina (OR 0,3; IC 95\% 0,18 a 0,50 p<0,01); sin embargo, al separar los estudios observacionales de los ensayos clínicos, el beneficio es evidente solo en los primeros, en tanto que en los últimos no hubo diferencias significativas (OR 0,49 ; IC 95\% 0,09-2,56, $p=0,40$ ). Fueron tres los ensayos clínicos incluidos en este metaanálisis:

1) El de Tolosana et al. ${ }^{16}$, se llevó a cabo con 101 pacientes y no reportó diferencias estadísticamente significativas para el desenlace sangrado cuando se comparó la terapia ininterrumpida con warfarina con la terapia puente con heparinas (OR: 1,02; IC 95\% 0,26-3,97; $p>0,9$ ).

2) El de Milic et al. ${ }^{17}$ fue diseñado para evaluar la efectividad de un sellante de fibrina para prevenir complicaciones hemorrágicas en pacientes que recibían terapia anticoagulante y serían llevados a implante de marcapasos.

3) El de Cheng et al. ${ }^{18}$, en el cual solo 7 de 100 pacientes incluidos recibieron terapia puente con heparinas, $y$ por tanto no permite inferir un resultado significativo al comparar la terapia ininterrumpida con warfarina con la terapia puente con heparinas. En este metaanálisis no se encontraron diferencias en el riesgo de eventos tromboembólicos entre las dos estrategias (OR 0,65; IC 95\% $0,14-3,0, p=0,58)^{14}$.

Los metaanálisis publicados en 2012 por Siegal et al. ${ }^{12}$ y por Bernard et al. ${ }^{13}$ obtuvieron calificación de calidad baja y muy baja, respectivamente. Incluyeron, en su mayoría, estudios observacionales, heterogéneos y no todos abarcaron población de alto riesgo embólico. En el de Bernard, datos de seis estudios reportaron que el 50,5\% de los pacientes fue clasificado en alto riesgo y el $17,2 \%$ en riesgo moderado. Aquellos que sí incluyeron población de alto riesgo, también están incluidos en el metaanálisis de Ghanbari et al. ${ }^{14}$

Recientemente Birnie et al., ${ }^{2}$ en su estudio Bruise Control, publicado en 2013, reafirmaron la conclusión del metaanálisis de Ghanbari et al. ${ }^{14}$, en un ensayo multicéntrico, aleatorizado, simple ciego y controlado, en el que compararon la warfarina ininterrumpida con la terapia puente con heparinas e incluyeron pacientes con riesgo de eventos tromboembólicos mayor o igual al 5\% por año (moderado y alto riesgo) que fueron sometidos a cirugía de implante de marcapasos o cardiodesfibriladores. El estudio terminó antes de lo previsto por recomendación del comité de seguridad al momento del segundo análisis interino cuando se documentó el beneficio de continuar la terapia con warfarina en lugar de efectuar la terapia puente. La incidencia de hematoma clínicamente significativo del bolsillo del dispositivo (aquel que requiriera cirugía, resultara en aumento de la estancia hospitalaria o precisara interrupción de la anticoagulación oral), fue menor en el grupo de warfarina ininterrumpida $(3,5 \%)$, en comparación con el grupo en quienes se realizó terapia puente con heparinas (16\%), (RR: 0,19; IC 95\% 0,10-0,36, p<0,001). Las complicaciones quirúrgicas mayores y tromboembólicas fueron poco frecuentes y no difirieron entre ambos grupos ${ }^{2}$. Para el desenlace sangrado, este ensayo clínico obtuvo una calificación metodológica alta, en tanto que para el desenlace de eventos tromboembólicos fue moderada debido a que este desenlace fue un objetivo secundario y el tamaño de la muestra podría no tener poder para encontrar diferencias, si existieran. Este es el único estudio que reporta resultados sobre satisfacción del paciente, mostrando diferencias estadísticamente significativas a favor de la terapia ininterumpida con warfarina $(\mathrm{p}<0,001)$.

Finalmente, Airaksinen et al. ${ }^{15}$, en el estudio FinPAC publicado en 2013, evaluaron la seguridad en un seguimiento a cuatro semanas, en términos de eventos de sangrado y tromboembólicos, de la interrupción de la anticoagulación oral con warfarina (dos días) comparada con la terapia de warfarina ininterrumpida en pacientes con alto riesgo embólico (score de $\mathrm{CHADS}_{2} \mathrm{VASC}_{2}$ de $3 \pm 1,6$, no portadores de válvulas mecánicas) que requerían implante de dispositivos de estimulación cardíaca, y reportaron que no existe diferencia estadísticamente significativa de riesgo de sangrado entre los dos grupos. En el grupo de terapia ininterrumpida se observó hematoma del bolsillo (cualquier sangrado) en el 33 vs. el $40 \%$ de los pacientes del grupo de terapia interrumpida. La estrategia de warfarina ininterrumpida no fue inferior a la de warfarina interrumpida con relación al desenlace sangrado (HR: 0,86; IC 95\% 0,66-1,12, $\mathrm{p}=0,001$ para no inferioridad) y no hubo necesidad de revisión del bolsillo cuando el implante se realizó con terapia de warfarina ininterrumpida. El desenlace hematoma mayor definido como cualquier sangrado o hematoma del bolsillo que requiriera intervención adicional o descontinuación de la anticoagulación, se presentó en seis casos en cada grupo (6 vs. 6\%). En el grupo de terapia interrumpida, un paciente presentó evento embólico (accidente cerebrovascular) tres días después del procedimiento y no se documentaron eventos tromboembólicos en quienes continuaron terapia de anticoagulación oral $^{15}$. Los investigadores concluyeron que el implante de dispositivos cardíacos puede realizarse de manera segura sin la descontinuación de la terapia con warfarina. El estudio tiene limitaciones en el tamaño de la muestra para detectar diferencias en el riesgo de sangrado mayor y eventos tromboembólicos. Estos análisis solo se pueden considerar descriptivos y exploratorios y no son suficientes para concluir no inferioridad o superioridad de una terapia respecto a la otra.

\section{Recomendaciones}

Después de revisar y evaluar la evidencia disponible hasta la fecha, el panel de expertos constituido por once profesionales de diferentes especialidades médicas (Cardiología, Electrofisiología, Epidemiología Clínica, Hemodinamia, Hematología, Medicina Interna y Medicina de Urgencias), estuvo a favor de la siguiente recomendación:

Continuar la anticoagulación oral con warfarina durante el perioperatorio de implante de dispositivos de estimulación cardíaca (marcapasos o cardiodesfibriladores implantables en pacientes con válvula cardíaca mecánica, fibrilación auricular o tromboembolia venosa y con riesgo moderado o alto para tromboembolia arterial (evidencia fuerte a favor).

Se emitieron además tres puntos de buena práctica clínica y una recomendación para investigación. 


\section{Puntos de buena práctica clínica}

El INR (del inglés International Normalized Ratio) debe mantenerse en rango terapéutico así:

- Un 2,5 a 3,5 para válvulas mecánicas en posición mitral.

- Entre 2,0 y 3,0 para válvulas mecánicas en posición aórtica, fibrilación auricular y tromboembolia venosa.

No se deben implantar dispositivos cardíacos en pacientes que ingieren dosis supraterapéuticas de warfarina.

Se recomienda que los pacientes sean incluidos en clínicas o programas de anticoagulación con el fin de recibir educación y de esta forma optimizar el control y seguimiento de la terapia anticoagulante, así como para identificar factores de riesgo (ej. polifarmacia), que puedan interferir con la terapia.

Se recomienda que los médicos que realizan implantes de dispositivos de estimulación cardíaca, en pacientes con moderado o alto riesgo tromboembólico tratados con anticoagulación oral con warfarina, optimicen esfuerzos para lograr una buena hemostasia durante el procedimiento.

\section{Recomendación para investigación}

Se requiere evidencia que evalúe los nuevos anticoagulantes en el manejo de la anticoagulación perioperatoria en pacientes con moderado y alto riesgo embólico que vienen siendo tratados con estas moléculas.

\section{Conclusiones}

La nueva evidencia permite modificar la recomendación propuesta por las guías SIGN y CHEST. La alternativa de continuar la terapia con warfarina en lugar de realizar terapia puente, debe considerarse en la práctica clínica.

\section{Actualización de la guía}

El grupo desarrollador actualizará esta guía cada tres años o antes si surge nueva evidencia que pueda modificar las recomendaciones. Se establecieron alarmas de búsqueda en las diferentes bases de datos. Cada autor revisará de forma independiente los nuevos experimentos o guías encontradas y finalmente se actualizarán en consenso las recomendaciones a las que haya lugar.

\section{Implicación sobre los recursos de la aplicación de las recomendaciones}

Se requiere el desarrollo de estudios económicos que aborden este tema. La implementación de la recomendación podría generar ahorro en el sistema de salud al reducir el riesgo de hematoma con el uso de terapia ininterrumpida con warfarina.

\section{Participación de los pacientes}

Durante el proceso de desarrollo de la guía no participaron pacientes. Sin embargo, en la reunión de consenso el grupo de expertos consideró los aspectos favorables para ellos al generar la recomendación. La ininterrupción de la terapia con warfarina simplifica el esquema de manejo para el paciente y facilita la continuidad de la terapia después del procedimiento.

\section{Participantes en el Consenso}

Alejandro Olaya.

Programa de Especialización en Cardiología de la Fundación Universitaria de Ciencias de la Salud. Cardiología y Electrofisiología Hospital de San José de Bogotá. Servicio de Electrofisiología Hospital Cardiovascular del Niño de Cundinamarca. Electrofisiología, Fundación Santa Fe de Bogotá.

Hernán J. Calvo.

Medicina Interna, Fundación Universitaria de Ciencias de la Salud.

Catalina del P. Pinzón.

Medicina Interna, Fundación Universitaria de Ciencias de la Salud.

Magda J. Alba.

Coordinación de Investigaciones Posgrados Medicina, Fundación Universitaria de Ciencias de la Salud.

Magda Cepeda.

División de investigaciones de posgrado, Fundación Universitaria de Ciencias de la Salud.

Guillermo Mora.

Cardiología y Electrofisiología, Fundación Santa Fe de Bogotá. Universidad Nacional de Colombia.

María H. Solano.

Medicina Interna y Hematología, Hospital de San José.

Medicina Interna y Hematología, Fundación Universitaria de Ciencias de la Salud.

John Liévano.

Cardiología y Hemodinamia, Hospital de San José. Fundación Universitaria de Ciencias de la Salud.

\section{Otros expertos participantes en el panel}

Claudia Ximena Clavijo.

Unidad de cuidados coronarios, Hospital de San José.

John Sprockel.

Fundación Universitaria de Ciencias de la Salud.

\section{Financiación}

Este trabajo fue financiado por el Servicio de Cardiología del Hospital de San José y la División de Investigaciones de la Fundación Universitaria de Ciencias de la Salud.

\section{Conflicto de intereses}

Los autores declaran no tener ningún conflicto de intereses.

\section{Bibliografía}

1. Mond HG, Proclemer A. The $11^{\text {th }}$ world survey of cardiac pacing and implantable cardioverter-defibrillators: calendar year 2009 -a World Society of Arrhythmia's project. PACE. 2011;34(8):1013-27. Publicación electrónica 29 Jun 2011. 
2. Birnie DH, Healey JS, Wells GA, Verma A, Tang AS, Krahn AD, et al. Pacemaker or defibrillator surgery without interruption of anticoagulation. New Eng J Med. 2013;368(22):2084-93. Publicación electrónica 11 May 2013.

3. Douketis JD, Spyropoulos AC, Spencer FA, Mayr M, Jaffer AK, Eckman $\mathrm{MH}$, et al. Perioperative management of antithrombotic therapy: Antithrombotic Therapy and Prevention of Thrombosis, $9^{\text {th }}$ ed: American College of Chest Physicians Evidence-Based Clinical Practice Guidelines. Chest. 2012;141 2 Suppl, e326S50S. Publicación electrónica 15 Feb 2012.

4. Jan Brozek AO, Holger Schünemann. GRADEpro. 3.2 for Windows ed. 2008.

5. Consortium ANS. El Instrumento AGREE II. Versión electrónica, 2009. Disponible en: http://www.agreetrust.org

6. SIGN SIGN. SIGN 50: a guideline developer's handbook2004 26/08/2013. Disponible en: http://www.sign.ac.uk/guidelines/ fulltext/50/

7. Pernod G, Godier A, Gozalo C, Tremey B, Sie P. French clinical practice guidelines on the management of patients on vitamin $\mathrm{K}$ antagonists in at-risk situations (overdose, risk of bleeding, and active bleeding). Thrombosis Research. 2010;126(3):e167-74. Publicación electrónica 16 Jul 2010.

8. Keeling D, Baglin T, Tait C, Watson H, Perry D, Baglin C, et al. Guidelines on oral anticoagulation with warfarin - fourth edition. Brit J Haematol. 2011;154(3):311-24. Publicación electrónica 16 Jun 2011.

9. Spyropoulos AC, Douketis JD. Guidelines for antithrombotic therapy: periprocedural management of antithrombotic therapy and use of bridging anticoagulation. International angiology: a journal of the International Union of Angiology. 2008;27(4):333-43. Publicación electrónica 5 Ago 2008.

10. Antithrombotics: indications and management [database on the Internet]. SIGN Publication No. 129. 2013 [consultado 26 Ago 2013]. Disponible en: http://www.sign.ac.uk

11. Jamula E, Douketis JD, Schulman S. Perioperative anticoagulation in patients having implantation of a cardiac pacemaker or defibrillator: a systematic review and practical management guide. JTH. 2008;6(10):1615-21. Publicación electrónica 22 Jul 2008.

12. Siegal D, Yudin J, Kaatz S, Douketis JD, Lim W, Spyropoulos AC. Periprocedural heparin bridging in patients receiving vitamin $\mathrm{K}$ antagonists: systematic review and meta-analysis of bleeding and thromboembolic rates. Circulation. 2012;126(13):1630-9. Publicación electrónica 23 Ago 2012.

13. Bernard ML, Shotwell M, Nietert PJ, Gold MR. Meta-analysis of bleeding complications associated with cardiac rhythm device implantation. Circ Arrhyth Electrophysiol. 2012;5(3):468-74. Publicación electrónica 27 Abr 2012.

14. Ghanbari H, Phard WS, Al-Ameri H, Latchamsetty R, Jongnarng$\sin \mathrm{K}$, Crawford T, et al. Meta-analysis of safety and efficacy of uninterrupted warfarin compared to heparin-based bridging therapy during implantation of cardiac rhythm devices. Am J Cardiol. 2012;110(10):1482-8. Publicación electrónica 22 Ago 2012.

15. Airaksinen KE, Korkeila P, Lund J, Ylitalo A, Karjalainen $P$, Virtanen V, et al. Safety of pacemaker and implantable cardioverter-defibrillator implantation during uninterrupted warfarin treatment-the FinPAC study. Int J Cardiol. 2013;168(4):3679-82. Publicación electrónica 16 Jul 2013.

16. Tolosana JM, Berne P, Mont L, Heras M, Berruezo A, Monteagudo J, et al. Preparation for pacemaker or implantable cardiac defibrillator implants in patients with high risk of thrombo-embolic events: oral anticoagulation or bridging with intravenous heparin? A prospective randomized trial. Eur Heart J. 2009;30(15):1880-4. Publicación electrónica 3 Jun 2009.

17. Milic DJ, Perisic ZD, Zivic SS, Stanojkovic ZA, Stojkovic AM, Karanovic ND, et al. Prevention of pocket related complications with fibrin sealant in patients undergoing pacemaker implantation who are receiving anticoagulant treatment. Europace. 2005;7(4):374-9. Publicación electrónica 10 Jun 2005.

18. Cheng A, Nazarian S, Brinker JA, Tompkins C, Spragg DD, Leng $\mathrm{CT}$, et al. Continuation of warfarin during pacemaker or implantable cardioverter-defibrillator implantation: a randomized clinical trial. Heart Rhythm. 2011;8(4):536-40. Publicación electrónica 15 Dic 2010. 